\title{
Shape and deformation measurements of rough surfaces by phase-shifting digital holography
}

\author{
Ichirou Yamaguchi \\ Scientist Emeritus of RIKEN, Higashi-Hisakata 3-4-32. Kiryu, Gunma 376-0053
}

Received November 14, 2021; accepted December 06, 2021; published December 31, 2021

\begin{abstract}
In digital holography recording the reconstruction of holograms is performed digitally by modern photonic devices to increase optical non-contacting measurements of various kinds of surfaces, both specular and rough. In this article we discuss these features of digital holography using phase shifting techniques that have greatly extended holographic capabilities.
\end{abstract}

Introducing a phase-shifting fringe pattern method of analysis into digital holography (DH) was an important step in its metrological applications. The basic setup for phase-shifting digital holography (PSDH) is modified by adding a PZT-mirror in the reference beam path as illustrated in Fig.1. The reference and object beam are travelling in the in-line configuration so the maximum bandwidth of object frequency can be captured. At least three holograms are acquired after stepwise phase shifts of the reference beam. The interference intensity detected by the $\mathrm{CCD}$ is given by:

$$
\begin{aligned}
I_{H}(x, y, \delta) & =\left|U_{R} \exp (i \delta)+U(x, y)\right|^{2}=\left|U_{R}\right|^{2}+|U|^{2}+ \\
& +2 \Re\left[U_{R} U^{*} \exp (i \delta)\right],
\end{aligned}
$$

where $U_{R}$ is the complex amplitude of the reference beam and $\delta$ is the phase difference between the object and the reference waves. By using the three phase-shifting procedure with a phase step of $\pi / 2$, we can derive the complex amplitude of the object wave such as $[1,2]$ :

$$
\begin{aligned}
U(x, y) & =\frac{1-i}{4 U_{R}^{*}}\left\{I_{H}(x, y, 0)-I_{H}\left(x, y, \frac{\pi}{2}\right)+\right. \\
& \left.+i\left[I_{H}\left(x, y, \frac{\pi}{2}\right)-I_{H}(x, y, \pi)\right]\right\}
\end{aligned}
$$

For analysis of image reconstruction, we adopt the coordinate system depicted in Fig. 2. The object wave at the CCD plane is represented as a Fresnel transform of the complex amplitude at the object plane $U(x, y)$ by:

$U_{I}(X, Y, Z)=\iint U(x, y) \exp \left[i k Z+i k \frac{(X-x)^{2}+(Y-y)^{2}}{2 Z}\right] d x d y$

where the integration is carried out over the area of CCD. We assume first a sufficient extension and ideal resolution of the device. Here the collimated reference beam is assumed with $z_{R}=\infty$. If we substitute Eq. (1) into Eq. (3), we find that the image plane is determined from the condition that the quadratic term of $x$ and $y$ in the exponent vanishes such as $z=-z_{o}$, where the complex amplitude becomes:

$U_{I}\left(X, Y,-z_{o}\right)=U_{o}(X, Y)$

if we neglect the limitation of finite size of CCD array.

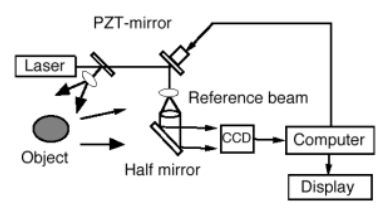

Fig. 1. Phase-shifting digital holography.

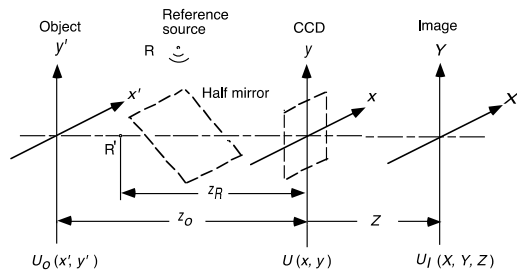

Fig. 2. Coordinate systems.

Image reconstruction (acc. Eq. (3)) can be numerically calculated by regarding it as either a Fourier transform or a convolution integral and replacing the integration by summation. In the first algorithm of reconstruction (the single FFT method), the sample interval of the image is given by $\lambda Z / L$, where $L$ is the size of CCD. The second algorithm, which uses the double FFT method, keeps the sampling interval equal to be the pixel pitch of CCD independent of the reconstruction distance. If we record an object by using a geometry shown in Fig. 2, the resolution is given by $\Delta x=\lambda z_{0} / L$, while the focal depth of the reconstructed image is given by $\Delta z=\lambda\left(z_{o} / L\right)^{2}$. The maximum object size to be recorded is equal to $N \Delta x$ with the pixel number $N$ along the $x$-direction in the single FFT algorithm, while it becomes equal to that of CCD in the double FFT method [3].

The phase to be detected by digital holography is given by the difference of the reconstructed complex amplitude that can be represented as follows [4]. The phase difference is due to changes of the incident angle or the wavelength of 
an object illumination beam. The wave vectors of this beam are represented by $\boldsymbol{k}_{a}$ and $\boldsymbol{k}_{b}$ as shown in Fig. 3(a). If we denote the vectors representing the observation direction by $\boldsymbol{k}_{a o}$ and $\boldsymbol{k}_{b o}$, the difference of the reconstructed phase corresponding to the identical points is given by:

$$
\begin{aligned}
\Phi(x, y) & =\left\langle\arg \left(U_{1}\right)-\arg \left(U_{2}^{*}\right)\right\rangle=\frac{\left\langle U_{1} U_{2}^{*}\right\rangle}{\sqrt{\left\langle I_{1} I_{2}\right\rangle}}= \\
& =-\left(k_{a z}-k_{b z}-k_{a o z}+k_{b o z}\right) h(x, y)-\left(k_{a z}-k_{b z}\right) x,
\end{aligned}
$$

where the incident plane is in the $x-z$ plane and the reference plane for the surface height is in the $x-y$ plane. In Eq. (5) the first term on the right-hand side means the phase difference proportional to the surface height and the second term stands for tilt components.

If we change the wavelength of the illumination from $\lambda_{a}$ to $\lambda_{b}$ and reconstruct each of the hologram with the same wavelength as in hologram recording, the phase difference is expressed with the incident angle $\theta_{s}$ and normal observation by:

$$
\begin{aligned}
\Phi(x, y) & =-\left(1+\cos \theta_{s}\right)\left(k_{a}-k_{b}\right) h(x, y)= \\
& =-2 \pi\left(1+\cos \theta_{s}\right) h(x, y) / \Lambda
\end{aligned}
$$

which means the contours of an object height with a sensitivity that is associated with so called synthetic wavelength given by

$$
\Lambda=1 /\left(1 / \lambda_{a}-1 / \lambda_{b}\right) .
$$

When the incident angle is changed from $\theta$ to $\theta+\Delta \theta$, the phase difference becomes:

$$
\begin{aligned}
\Phi(x, y) & =-k[\cos \theta-\cos (\theta+\Delta \theta)] h(x, y)-k[\sin \theta-\sin (\theta+\Delta \theta)] x= \\
& =2 k h(x, y) \sin \left(\theta+\frac{\Delta \theta}{2}\right) \sin \frac{\Delta \theta}{2}+2 k x \cos \left(\theta+\frac{\Delta \theta}{2}\right) \sin \frac{\Delta \theta}{2} \approx \\
& \approx \sim k h(x, y) \Delta \theta \sin \theta+k x \Delta \theta \cos \theta,
\end{aligned}
$$

where the lastline results from an approximation for $\Delta \theta$ being much smaller than $\theta$. This phase distribution is just equal to the phase of the projected fringes produced by the two coexisting beams. For small $\Delta \theta$ the height sensitivity of the phase difference is given by $\lambda / \Delta \theta \sin \theta$. The resultant distributions of the phase before and after the mirror rotation are subtracted from each other to produce the phase $\bmod (2 \pi)$. After phase-unwrapping we obtain the phase distribution that is given by Eq. (8). Next the tilt component is subtracted to provide surface height distribution from the reference plane. (a)

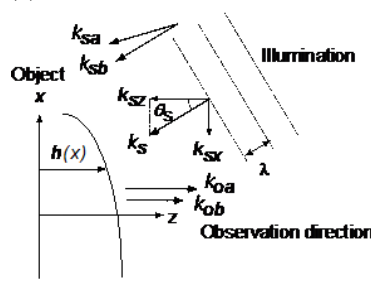

(b)

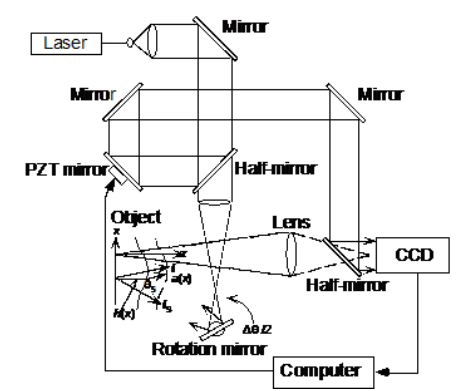

Fig. 3. (a) Principles and (b) setup of contouring.
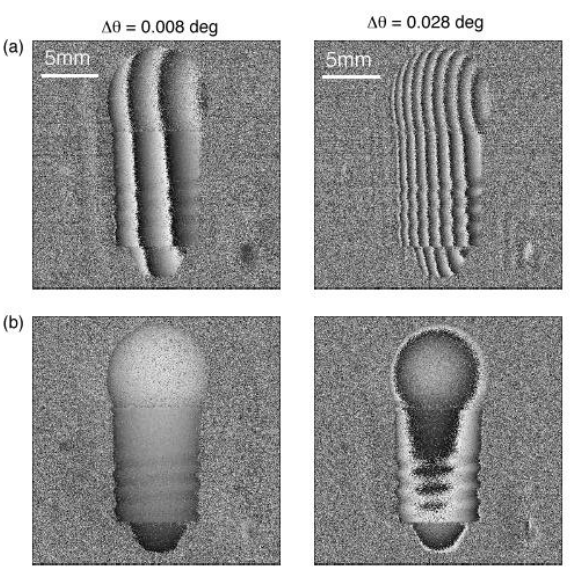

Fig. 4. Contours of a miniature bulb for different tilt angles.

Figure 4 represents the result obtained from the shape measurement of a miniature bulb painted white. The distributions of the phase difference shown in (a) contain the carrier component corresponding to the second term of the right-hand side of Eq. (8). By subtracting this component from the phase difference at $1024 \times 1024$ pixels before phase-unwrapping we align the reference plane parallel to the object plane as shown in Fig. 4(b). The resultant distribution contains noise associated with speckles. We suppressed this noise by extracting one point from each $2 \times 2$ matrix where the modulus of the product $\left|U_{1} U_{2}{ }^{*}\right|$ becomes maximum. This filtering is based on the fact that the phase value is more reliable for higher amplitude. The compressed data are then smoothed by averaging over each $2 \times 2$ matrix with final data pixels of $256 \times 256[5]$. The 3D maps and the cross-sections through the bulb axis before (a) and after the filtering (b) are shown in Figures 5(a) and (b). This method of surface shape measurement could be easily applied to small objects by employing a microscope system mentioned above.

In the out-of-plane deformation measurement $[6,7]$ the illumination is fixed and the phase difference of the reconstructed waves before and after object deformation 


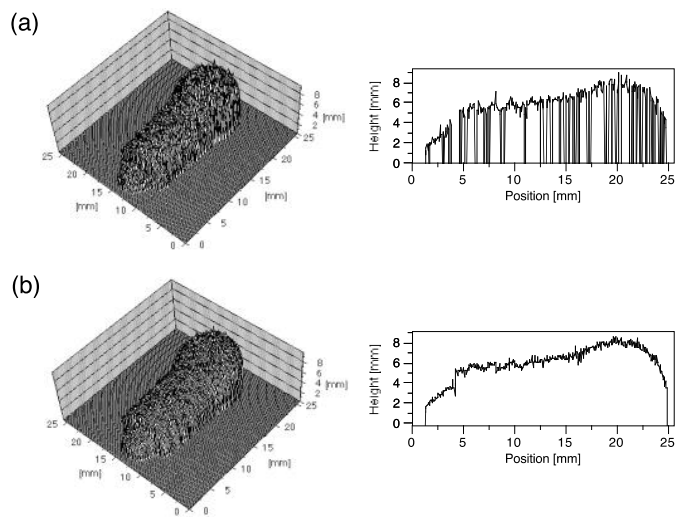

Fig. 5. Results from a miniature bulb under exponential filtering.

that depends on the vectors illustrated in Fig. 6. We denote the object displacement vector of the point $(x, y)$ by $\boldsymbol{a}(x, y)$. If the speckle displacement is much smaller than their mean size, the phase difference becomes:

$$
\Phi(x, y)=-\left(k_{s}-k_{o}\right) \cdot a(x, y) \text {. }
$$

This condition means that the systematic phase change can be observed as a result of cancellation of a random phase variation between different speckles. It limits the measurement of in-plane displacement. To measure both surface shape and deformation of diffusely reflecting surfaces we built the optical system shown in Fig. 7. We employed a setup for image plane hologram so that limitation on the object size can be relaxed while ensuring high light flux incident on the CCD. The surface shape is measured by tilting the mirror for object illumination. This setup is the same as that of phase-shifting electronic speckle interferometry (ESPI), but the DH can also be used for 3D objects by virtue of numerical focusing. We need not refocus the imaging lens on the position of interest and have only to record the hologram once before and after object deformation and mirror tilt. We also have much more freedom of suppression of speckle noise because the phase difference is directly derived instead of correlation fringes detected in ESPI as mentioned above.

The results of experiments conducted for out-of-plane deformation of a square plate of aluminum with the size of $50 \times 50 \mathrm{~mm}^{2}$ and $1 \mathrm{~mm}$ thick are shown in Fig. 8. The plate was pushed at its center while being supported at the circular edge. The phase difference (Fig. 8(a)) is the contour of out-of-plane displacement. The surface shape after total loading repeated 20 times is represented. The resolution is estimated to be $0.01 \mu \mathrm{m}$. For contouring a tilt of $\Delta \theta=0.25$ degrees was added to $\theta_{\mathrm{s}}=45 \mathrm{deg}$. After the removal of a carrier component the contours of (b) were obtained while (c) is the cross-section along the line indicated. The fluctuation of height is about $10 \mathrm{~nm}$.
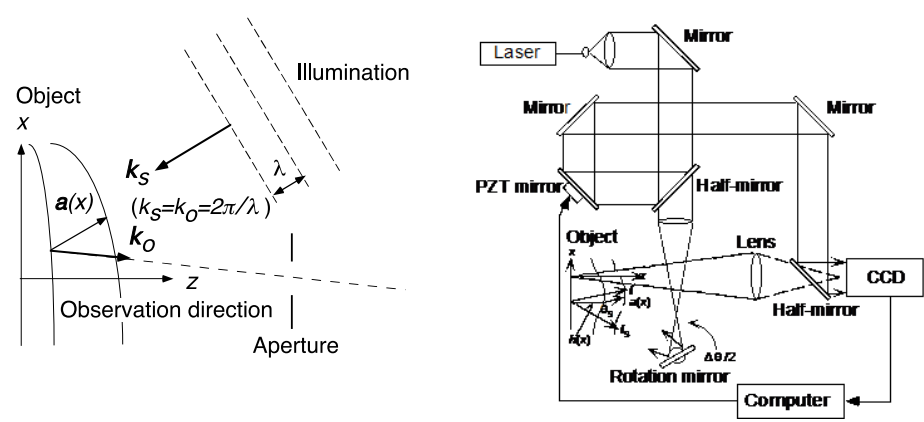

Fig.6. Principles of deformation measurement.

Fig. 7. Setup of deformation measurement.

which is an order of surface roughness of the specimen. Vibration analysis has also been conducted by time averaging the hologram intensity during the sinusoidal vibration over the exposure time much longer than the vibration period. At least three phase shifted holograms are recorded and reconstructed. The reconstructed image is modulated by the 0 -order Bessel function, as in the classical holographic vibration analysis [8].
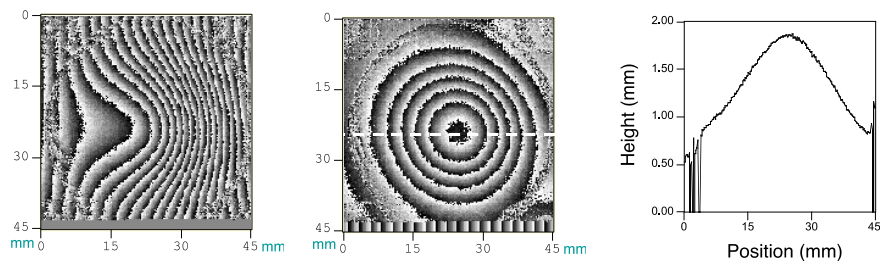

Fig. 8. (a) Phase deformation, (b) Removed tilt, (c) Cross-section along the line

In this article we surveyed the principle and applications of phase-shifting digital holography that has improved performances of DHsubstantially. Since it delivers values of complex amplitude at the CCD plane directly without any filtering process, both derivation and processing of required information on the shape and deformation are straightforward and simple. A full number of CCD pixels is utilized for the final image with a quality much better than in the off-axis setup. We also proposed a new method of phase analysis with higher performance.

\section{References}

[1] G. Bruning, D.R. Herriott, J.E. Gallagher, D.P. Rosenfeld, A.D. White, D.J. Brangaccio, Appl. Opt. 13, 2693 (1974).

[2] I. Yamaguchi, T. Zhang, Opt. Lett. 22, 1268 (1997).

[3] F. Zhang, I. Yamaguchi, L.P. Yaroslavsky, Opt. Lett. 29, 1668 (2004).

[4] I. Yamaguchi, Optics and Lasers in Engineering 39, 411 (2003).

[5] I. Yamaguchi, M. Yokota, Opt. Eng. 48085602 (2009).

[6] I. Yamaguchi, J. Kato, S. Ohta, Opt. Rev. 8, 85 (2001).

[7] I. Yamaguchi, J. Kato, H. Matsuzaki, Opt. Eng. 42, 1267 (2003).

[8] F. Zhang, J.D.R. Valera, I. Yamaguchi, M. Yokota, G. Mills, Opt. Rev. 11, 5 (2004). 